\title{
Research on a Numerical Calculation for Ball Bearings Based on a Finite Initial Value Search Method
}

\author{
Jing Hu $\mathbb{i}$, , ${ }^{1,2}$ XiaoLi Qiao $\mathbb{D}$, ${ }^{1,2}$ QiongYing Lv $\mathbb{D}^{1,2}$ XinMing Zhang $\mathbb{D}^{1,2,3}$ \\ and XiaoPing Zhou iD ${ }^{1,2}$ \\ ${ }^{1}$ Changchun University of Science and Technology, No. 7089 Satellite Road, Changchun, Jilin, China \\ ${ }^{2}$ Ministry of Education Key Laboratory for Cross-Scale Micro and Nano Manufacturing, Changchun 130022, China \\ ${ }^{3}$ Foshan University, Foshan, Guangdong, China \\ Correspondence should be addressed to XinMing Zhang; zxmcust163@163.com
}

Received 6 October 2020; Revised 4 December 2020; Accepted 18 December 2020; Published 4 January 2021

Academic Editor: Denis Benasciutti

Copyright $(92021$ Jing Hu et al. This is an open access article distributed under the Creative Commons Attribution License, which permits unrestricted use, distribution, and reproduction in any medium, provided the original work is properly cited.

To address the disadvantages of the traditional solution method of the quasistatic ball bearing model, which requires the acquisition of the initial value with experience, this paper proposes a new method for a finite initial value optimization to solve the quasistatic ball bearing model. A bilevel solution model is established; the first layer is the initial value strategy model, and the second layer is the numerical optimization model. The Levenberg-Marquardt algorithm and the Armijo algorithm are adopted to solve the model. The correctness of the new method is proved by a case study of calculating a parameter of the bearing compared with the traditional method. The experiment results show that the proposed method can realize the fast and finite solution of the quasistatic ball bearing model. Additionally, the new method can be extended to the calculation of the double-decker ball bearings. It provides a reasonable and effective way for the exploration of the initial value problem of the solution of a quasistatic ball bearing model.

\section{Introduction}

Ball bearings are indispensable core components of mechanical equipment, and the numerical calculation of their mechanical models forms the basis for exploring bearing characteristics [1-3]. The history of mechanical analysis models of ball bearings can be categorized into four stages, a static analysis model [4], a quasistatic analysis model [5], a quasidynamic model [6], and a dynamic analysis model [7]. After the emergence of the classic model, many scholars have established some models based on these classical theories as follows: in terms of life span research, such as a fatigue damage accumulation rating life model [8] and a dynamic contact wear model [9]; in terms of lubrication, such as an EHL and quasidynamic numerical coupled analysis model $[10,11]$; in terms of structural design, such as a bionic design model [12]; in terms of dynamics, such as a ball dynamics model [13], a sliding model [14, 15], a kinematics mathematical model [16], a raceway free control model [17], and under the arbitrary force quasidynamic model [18]; and in the aspect of noise research, such as a radiation noise calculation model [19]. When exploring the potential bearing performance, scholars have not been satisfied with the structure of single-layer bearings. Anderson first proposed the design concept of double-decker bearings [20], and then some scholars carried out a series of research on double-decker bearings. For example, the numerical analysis on the double-decker rolling bearing is studied [21], the mechanical model of a "Z-" type and "I-" type doubledecker ball bearing is established $[22,23]$. In the above studies, the traditional Newton-Raphson algorithm is applied to most of the numerical model solutions for ball bearings. However, the Newton-Raphson algorithm is dependent on the initial value; the iterative initial value is very sensitive, easily falling into a local optimal solution, and it does not have global convergence. For strong nonlinear equations, the possibility of multiple sets of solutions exists in theory. It is often necessary to experiment with different initial estimates, and the number of parameters in quasistatic equations is large; thus, the difficulty of choosing an initial value is drastically increased, which presents a major problem during the numerical calculation. 
Compared with the research using numerical analysis models, only a few studies assess the initial value of the numerical calculation model. Wang et al. constrained the unknowns of the nonlinear equations and improved the nonconvergence problem caused by the large deviation between the initial value and the result of the Newton-Raphson method in solving the nonlinear equations [24]. However, only the constraints of the initial value are given; the initial search point is not given, and the deterministic search algorithm is not given. Zhang et al. established the relationship between load and displacement in the state of dry friction and proposed a fast and reliable energy method based on the principle of minimum potential energy to solve the initial value of the bearing displacement in the state of dry friction [25]. However, the influence of inertial force and gyroscopic moment is ignored when the relation of load-displacement is established, so it is not suitable for high-speed calculations.

A lot of studies have shown that many engineering problems can be solved as optimization problems [26, 27]. Therefore, to address these problems, a finite initial value search method is proposed based on extensive research into the problem of solving a quasistatic ball bearing model under radial and axial forces conditions.

The main contributions of this paper are as follows:

(1) A bilevel solution model based on the initial value strategy model and the numerical optimization model is established. The Levenberg-Marquardt algorithm and the Armijo algorithm are adopted to solve the model and to realize a fast and finite solution.

(2) In the proposed model, the initial value search strategy is given; thus, solving the disadvantages of the traditional analysis method requires the acquisition of the initial value with experience.

(3) The new method can be extended to the calculation of double-decker ball bearings, which improves the applicability of the method. It provides a new way for solving the initial value problem of the doubledecker bearing numerical model.

The rest of this paper is organized as follows. Section 2 analyzes the quasistatic models of the single-layer ball bearing and the double-decker ball bearing, which provides the basis for the following contents. In Section 3, a bilevel solution model for the quasistatic solution of ball bearings is established, and the experiment is carried out based on the proposed method to verify its validity and applicability. In Section 4, an important parameter of bearing contact angle is selected as the case study and compared with the traditional method to verify the correctness of the new method. The comparison of the search step, the offset distance, and the computation time is given. Section 5 concludes the paper.

\section{Quasistatic Model}

2.1. Analysis Model of Single-Layer Ball Bearing. An analysis model of the single-layer ball bearing is set up, and the location diagram of the $i$ th ball of the single-layer ball bearing and the roll gap curvature center is shown in Figure 1(a). The outer ring of the single-layer ball bearing is fixed on a bearing pedestal, so the center of the outer ring raceway curvature $\mathrm{O}_{o 2 n}$ is considered fixed before and after loading, the inner ring raceway curvature center position changes from $O_{o 1 n}$ to $\mathrm{O}_{o 1 n}^{\prime}$, and the ball center position changes from $O_{\mathrm{o} i}$ to $\mathrm{O}_{o i}^{\prime}$.

Assuming that the single-layer bearing is subject to a radial force $F_{r}$ and an axial force $F_{a}$, the $i$ th ball of the singlelayer bearing is subjected to a centrifugal force $F_{i}$. The forces on the ball at the inner and outer layers of the bearing are shown in Figure 1(b). The forces in contact with the inner and outer rings are $Q_{1 i}$ and $Q_{2 i}$. The gyroscopic moment is $M_{i}$.

According to Figure 1(a), at any position angle, the distance between the curvature center of the outer ring and the ball center is calculated as shown in the following equations:

$$
\begin{aligned}
& A_{2 i}=X \sin \alpha_{0 i}+\lambda_{a}-\Delta_{a}, \\
& B_{2 i}=X \cos \alpha_{0 i}+\left(\lambda_{r}-\Delta_{r}\right) \cos \varphi_{i} .
\end{aligned}
$$

The geometric relationships are shown in the following equations according to the Pythagorean theorem [27]:

$$
\begin{aligned}
\lambda_{2 i}= & \sqrt{A_{1 i}^{2}+B_{1 i}^{2}}-\left(f_{o}-0.5\right) D_{b}, \\
\lambda_{1 i}= & \sqrt{\left(A_{2 i}-A_{1 i}\right)^{2}+\left(B_{2 i}-B_{1 i}\right)^{2}} \\
& -\left(f_{i}-0.5\right) D_{b} .
\end{aligned}
$$

According to Figure 1(b), the force balance equations of the $i$ th ball are shown as follows:

$$
\begin{array}{r}
Q_{1 i} \sin \alpha_{1 i}-Q_{2 i} \sin \alpha_{2 i}-\frac{M_{i}}{D_{b}} \\
\cdot\left(\xi_{1 i} \cos \alpha_{1 i}-\xi_{2 i} \cos \alpha_{2 i}\right)=0, \\
Q_{1 i} \cos \alpha_{1 i}+F_{i}-Q_{2 i} \cos \alpha_{2 i}+\frac{M_{i}}{D_{b}} \\
\cdot\left(\lambda_{1 i} \sin \alpha_{1 i}-\lambda_{2 i} \sin \alpha_{2 i}\right)=0
\end{array}
$$

When the force of the ball bearing is balanced, the force equilibrium equations of the inner raceway can be represented as follows:

$$
\begin{array}{r}
\sum_{i=1}^{Z}\left[Q_{1 i} \cos \alpha_{1 i}+\frac{\xi_{1 i} M_{i}}{D_{b}} \sin \alpha_{1 i}\right] \cos \varphi_{i}=F_{r}, \\
\sum_{i=1}^{Z}\left[Q_{1 i} \sin \alpha_{1 i}-\frac{\xi_{1 i} M_{i}}{D_{b}} \cos \alpha_{1 i}\right]=F_{a} .
\end{array}
$$

The contact force between the ith ball of the single-layer bearing and the inner and outer rings can be expressed by the following equation, where $K$ is the contact stiffness between the ball and the inner and outer rings:

$$
\begin{aligned}
& Q_{1 i}=K_{1 i} \lambda_{1 i}^{1.5}, \\
& Q_{2 i}=K_{2 i} \lambda_{2 i}^{1.5} .
\end{aligned}
$$




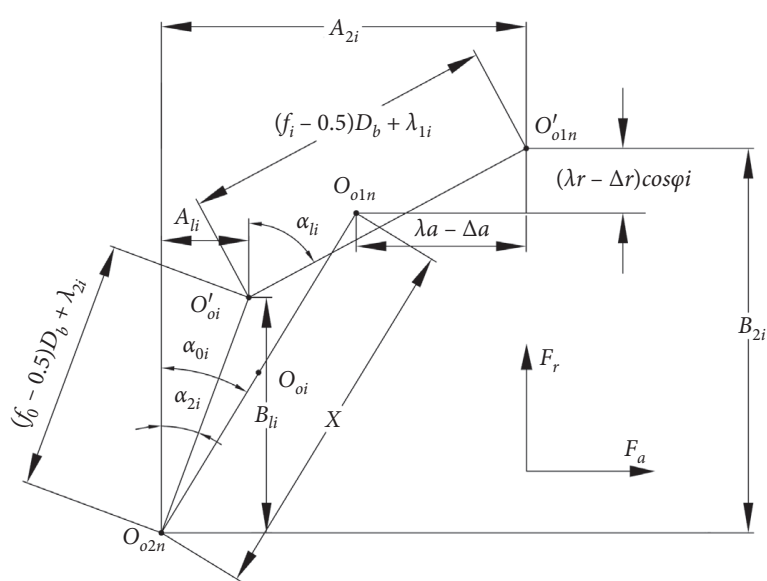

(a)

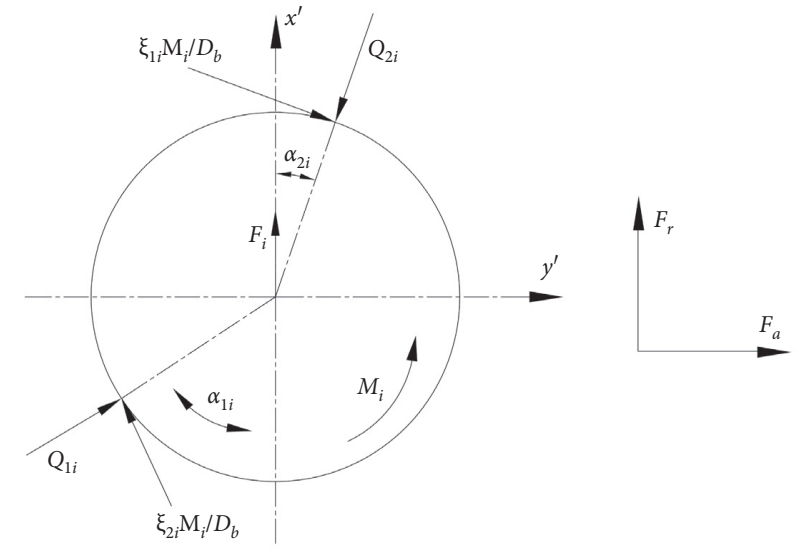

(b)

Figure 1: Analysis model of the single-layer ball bearing [28]. (a) The relationship diagram of the position of the $i$ th ball center and the curvature center of the raceway for the single-layer ball bearings. (b) The force diagram of the single-layer ball bearing.

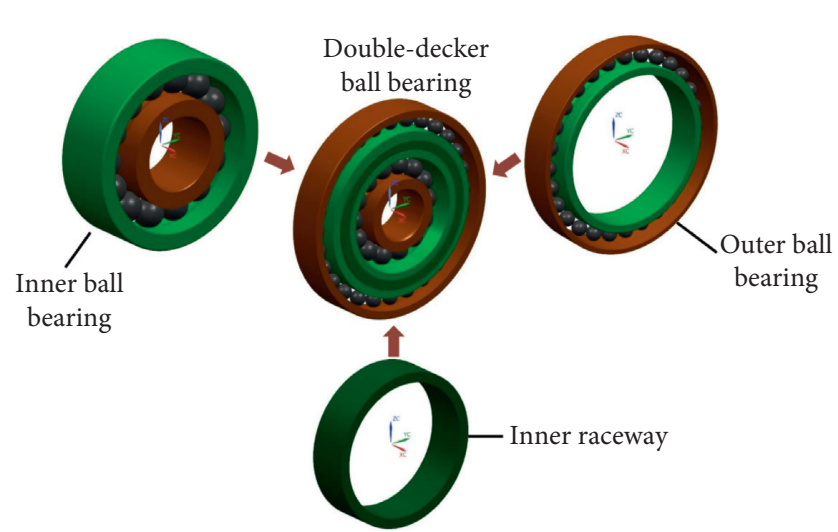

FIGURE 2: The structure of the double-decker ball bearing.

2.2. Analysis Model of Double-Decker Ball Bearing. A doubledecker ball bearing is composed of two single ball bearings, and its structural diagram is shown in Figure 2.

The relevant geometric parameters of the double-decker ball bearing are shown in Figure 3. The formula of the numerical analysis model is similar to that of the single-layer bearing, with a difference occurring in the middle ring balance formula, as shown in equations (11) and (12) [29].

$$
\begin{gathered}
\sum_{n=1}^{Z_{o b}}\left[Q_{o 1 n} \cos \alpha_{o 1 n}+\frac{\xi_{o 1 n} M_{o n}}{D_{o b}} \sin \alpha_{o 1 n}\right] \cos \varphi_{o n} \\
=\sum_{m=1}^{Z_{i b}}\left[Q_{i 2 m} \cos \alpha_{i 2 m}+\frac{\xi_{i 2 m} M_{i m}}{D_{i b}} \sin \alpha_{i 2 m}\right] \cos \varphi_{i m} \\
\sum_{n=1}^{Z_{o b}}\left[Q_{o 1 n} \sin \alpha_{o 1 n}-\frac{\xi_{o 1 n} M_{o n}}{D_{o b}} \cos \alpha_{o 1 n}\right] \\
=\sum_{m=1}^{Z_{i b}}\left[Q_{i 2 m} \sin \alpha_{i 2 m}-\frac{\xi_{i 2 m} M_{i m}}{D_{i b}} \cos \alpha_{i 2 m}\right]
\end{gathered}
$$

\section{The Proposed Method}

3.1. Algorithm Selection. Common optimization algorithms include heuristic algorithms and accurate algorithms [30, 31]. The Newton-Raphson method is one of the accurate algorithms, which is widely used in the numerical calculation of ball bearings, and although the algorithm reserves the first- and second-order Taylor series and quadratic convergence speed, every step needs to compute the Hessian matrix, and the computation is complex. To improve the efficiency, the Gauss-Newton algorithm and the Levenberg-Marquardt algorithm were introduced. However, the disadvantage of the Gauss-Newton algorithm is that it cannot be iterated when the Hessian matrix is not satisfied with the rank. The Levenberg-Marquardt algorithm can address the Hessian matrix that is not being satisfied with rank or not being positive, which is an improvement on the Newton-Raphson method. The Levenberg-Marquardt algorithm is one of the optimization algorithms [32, 33]. It is a nonlinear optimization method combining the Gauss-Newton method and the gradient descent method. It uses standard numerical optimization calculation and it is a fast algorithm. But the step size needs to be calculated in the process of solving, and the Armijo algorithm is a common line search method to find step length in solving nonlinear optimization problems [34]. Therefore, the Levenberg-Marquardt algorithm and the Armijo algorithm are adopted in this paper to solve nonlinear equations.

\subsection{Establishment of the Bilevel Solution Model}

3.2.1. The First-Layer Model. The first-layer model is the initial value search model. The contact deformation value $\lambda_{1 i}$ and $\lambda_{2 i}$ of the bearing should be greater than zero in the actual working condition. In the solution of nonlinear equations, if the initial value is not selected properly, the contact deformation of the solution will be less than zero, and then the results are not consistent with the actual situation. So, the numerical relation of equations (3) and (4) are converted into 


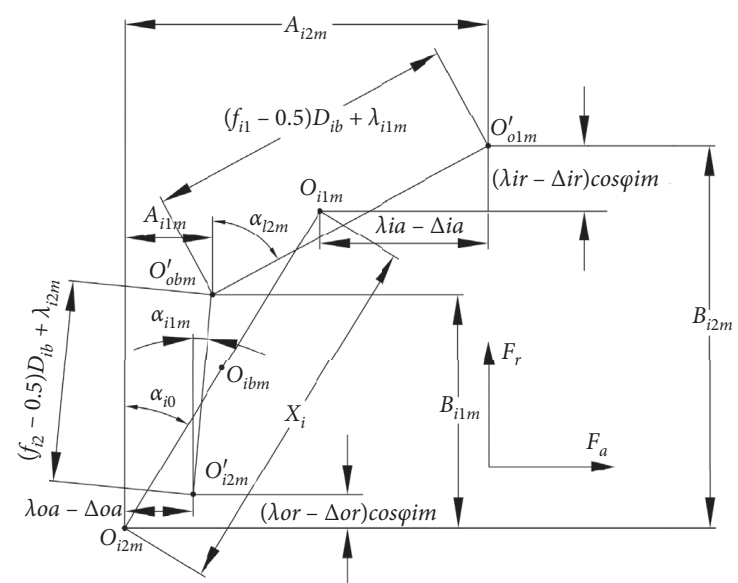

(a)

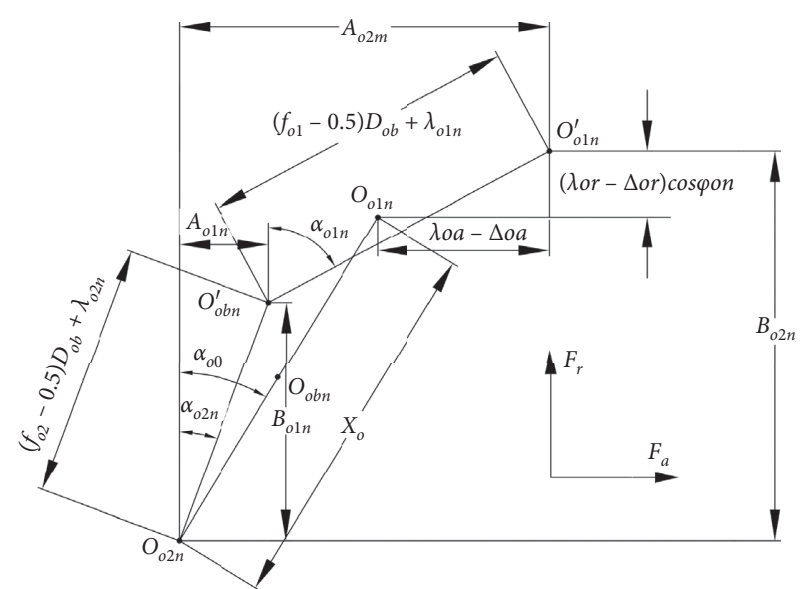

(b)

FIGURE 3: Relation diagram of the position of the $m$ th and $n$th ball centers and the raceway curvature centers of the inner and outer of the double-decker ball bearings.

two circles for analysis. The contact deformation equations (3) and (4) can be regarded as two circles: one with $(0,0)$ as the center and $\left(f_{o}-0.5\right) D_{b}$ as the radius and the other with $\left(A_{1 i}\right.$, $\left.B_{1 i}\right)$ as the center and $\left(f_{i}-0.5\right) D_{b}$ as the radius $[24,35]$, as shown in the black circle and the red circle of Figure 4.

According to the analysis of the relationship between the changes of the curvature center of the bearing before and after loading, the difference of the given initial value points can make the positions of the two circles exist in the following two relationships: intersection or separation, as the position of the black circle and the red circle shown in Figure 4. The initial point can fall into the region represented by the point of $M_{1}, M_{2}, M_{3}$, and $M_{4}$. According to equations (3) and (4), if the displacements $\lambda_{1 i}$ and $\lambda_{2 i}$ are greater than 0 , the position of the initial value point needs to fall outside the two circles.

In the actual running process of the ball bearing, the contact angle between the ball and the inner raceway gradually increases, while the contact angle between the ball and the outer raceway gradually decreases, so the slope of the line between the initial value and the center of the circle $O_{1}$ should be less than the slope of the line between the two centers.

According to the above analysis, three rules should be met in the solution process:

Rule $a$. The contact deformation value $\lambda_{1 i}$ and $\lambda_{2 i}$ should be greater than zero.

Rule $b$. The initial point needs to fall outside the two circles.

Rule $c$. The slope of the line between the initial value and the center of the circle $O_{1}$ should be less than the slope of the line between the two centers.

If the initial value makes the two circles tangent, there exists the situation that the string is searched along the tangent line to the intersection of two circles, but the value is negative, and the initial value needs to be adjusted again. If the two circles are separated by the initial value, there exists the situation that the given initial value is large, the two circles are far apart, and the number of search steps increases.

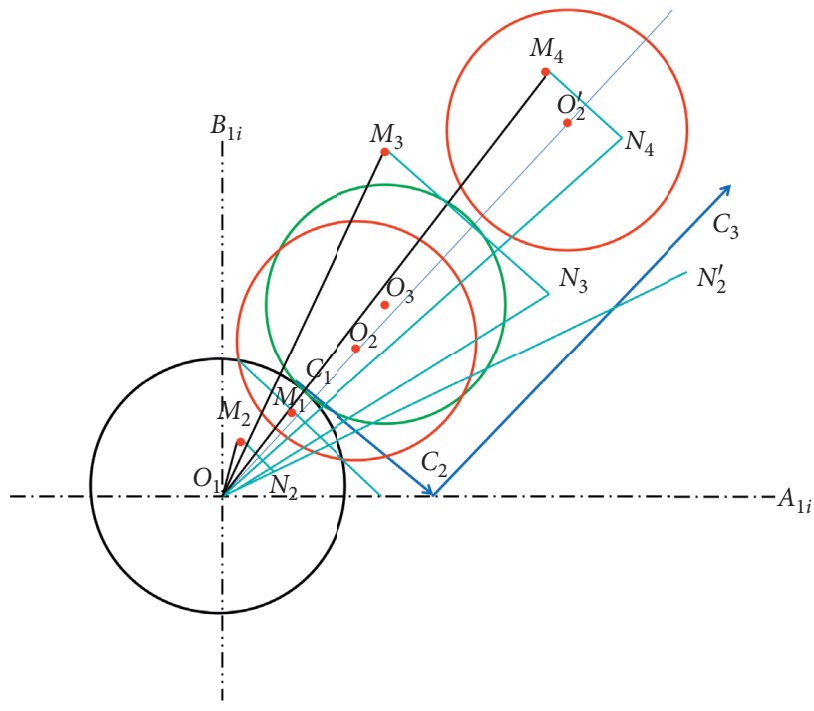

FIGURE 4: The relationship of initial values for numerical analysis of the single-layer ball bearings.

All of the above are initial value uncertainty problems. Based on the geometric equation of the change of the curvature center of the bearing and the changing trend of the contact angle between ball and raceway in the actual operation of the bearing, the initial value search model of the nonlinear equations is established to transform the uncertainty problem into the determination problem to enhance the stability of the initial value search.

As mentioned above, the initial value search strategy is established as follows:

(1) Simplify the circle $\mathrm{O}_{2}$. The intersection coordinate of the simplified model $O_{3}$ and the tangent circle $O_{1}$ is proposed as the initial value search point. Since there is no axial movement or radial movement of the bearing in the initial state and the bearing clearance is ignored in the current state, the intersection coordinates can be obtained by solving the equations of 
the two circles, as shown in Figure 4. In equations (1) and (2), $\lambda_{r}$ and $\lambda_{a}$ are the radial and axial displacement of the inner ring under the action of bearing external load, and $\Delta_{r}$ and $\Delta_{a}$ are radial and axial clearance. Because the initial state of the bearing is not under external load, $\lambda_{r}$ and $\lambda_{a}$ are zero, suppose ignoring it; $\Delta_{r}, \Delta_{a}$, and $X$ can be calculated according to the initial parameters of the bearing. According to equations (1) and (2) to find $A_{2 i}$ and $B_{2 i}$, substitute into equations (3) and (4) to calculate the intersection of the two circles.

(2) Plan the search path. As described above, the slope of the line between the initial value and the origin should be greater than the slope of the line between the center of the two circles, so search along the tangent direction to ensure that the search point is outside the two circles. If the initial value of convergence is not found, search the initial value along the upwards of the perpendicular direction of the tangent line.

The basis for establishing the above search strategy is as follows: the position diagram of the simplified model $\mathrm{O}_{3}$ and the real model $\mathrm{O}_{2}$ are shown in Figure 5. Suppose that the red circle $\mathrm{O}_{2}^{\prime}$ is the true position diagram of the bearing under force. As described above, the search for the initial values satisfying the nonlinear equations should be within the shadow range of the graph; the green circle $\mathrm{O}_{3}$ is a simplified model. According to equations (1)-(4), the simplified model $\mathrm{O}_{3}$ only changes the position of the center of the circle, while the radius remains unchanged. The simplified model $\mathrm{O}_{3}$ is tangent to the circle $O_{1}$, and the limit position is shown in the green circle $\mathrm{O}_{3}$ and circle $\mathrm{O}_{3}^{\prime}$ in Figure 5. It can be seen from the figure that, although the model is simplified, the search path proposed above is still in the shaded region, and the initial value is the value near the truth value. Therefore, the simplified model can be used as the initial search point to search the initial value. The uncertain initial search point is converted into a certain initial search point, which does not depend on the working condition and does not need to change the initial value manually.
The center of a circle $O_{1}$ is $(0,0)$, the center coordinate of the simplified model $\mathrm{O}_{3}$ is $\left(A_{1 i 3}, B_{1 i 3}\right)$, and the point of intersection is $(A, B)$. The line equation between the circle $O_{1}$ and the point of intersection is shown as follows:

$$
B_{1 i}=\frac{B_{1 i 3}}{A_{1 i 3}} A_{1 i} \text {. }
$$

The tangent equation is shown as follows:

$$
B_{1 i}^{\prime}=-\frac{A_{1 i 3}}{B_{1 i 3}} A_{1 i}^{\prime}+B+\frac{A_{1 i 3}}{B_{1 i 3}} A .
$$

The perpendicular equation of the tangent line is shown as follows:

$$
B_{1 i}^{\prime \prime}=\frac{B_{1 i 3}}{A_{1 i 3}} A_{1 i}^{\prime \prime}+\frac{A A_{1 i 3} B_{1 i 3}+B B_{1 i 3}^{2}}{A_{1 i 3}^{2}} .
$$

Then, the initial value search path is shown as follows:

$$
B_{1 i}=B_{1 i}^{\prime}+B_{1 i}^{\prime \prime} \text {. }
$$

Remark 1. Based on the analysis of the influence of initial value on the calculation results, an initial value search strategy is established. The intersection point between the simplified model $O_{3}$ and the circle $O_{1}$ is proposed as the initial search point. The search method of the initial value is given. Transform the initial uncertainty problem into a certainty problem. Thus, the problem of trying to find the initial value by manual experience is solved

3.2.2. The Second-Layer Model. The second layer establishes a numerical optimization model for the ball bearings, which transforms the problem of solving the nonlinear equations into an optimization problem. The optimization objective function is established as follows:

$$
\begin{aligned}
& \min F(\Omega) \\
& \text { s.t } \lambda_{1 i}>0, \lambda_{2 i}>0,
\end{aligned}
$$

where $\Omega$ is composed of $2 Z+2$ nonlinear equations, whose composition is shown as follows:

$$
\begin{aligned}
& F=\left[F_{1}, F_{2}, F_{3}, F_{4}\right] \text {, } \\
& \text { Where : }\left\{\begin{array}{l}
F_{1}=Q_{1 i} \sin \alpha_{1 i}-Q_{2 i} \sin \alpha_{2 i}-\frac{M_{i}}{D_{b}}\left(\xi_{1 i} \cos \alpha_{1 i}-\xi_{2 i} \cos \alpha_{2 i}\right), \\
F_{2}=Q_{1 i} \cos \alpha_{1 i}+F_{i}-Q_{2 i} \cos \alpha_{2 i}+\frac{M_{i}}{D_{b}}\left(\lambda_{1 i} \sin \alpha_{1 i}-\lambda_{2 i} \sin \alpha_{2 i}\right), \\
F_{3}=F_{r}-\sum_{i=1}^{Z}\left[Q_{1 i} \cos \alpha_{1 i}+\frac{\xi_{1 i} M_{i}}{D_{b}} \sin \alpha_{1 i}\right] \cos \varphi_{i}, \\
F_{4}=F_{a}-\sum_{i=1}^{Z}\left[Q_{1 i} \sin \alpha_{1 i}-\frac{\xi_{1 i} M_{i}}{D_{b}} \cos \alpha_{1 i}\right],
\end{array}\right. \\
& i=1 \sim z \text {. }
\end{aligned}
$$




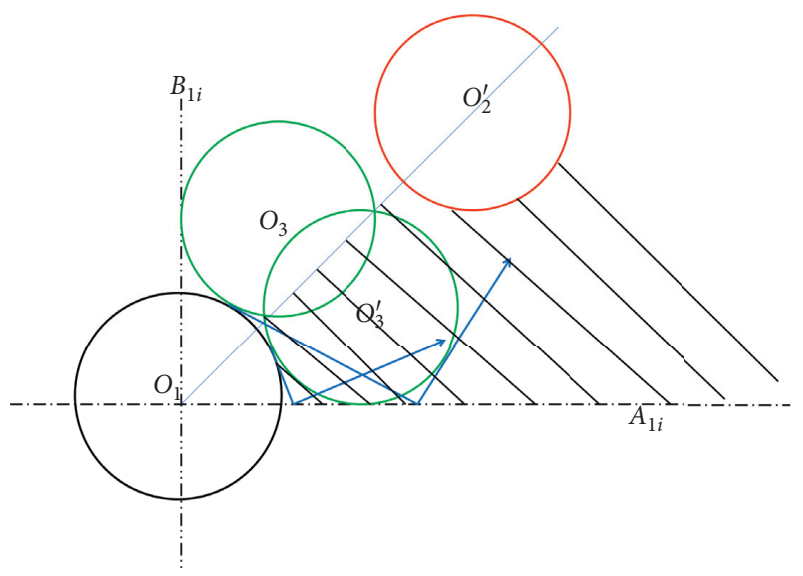

(a)

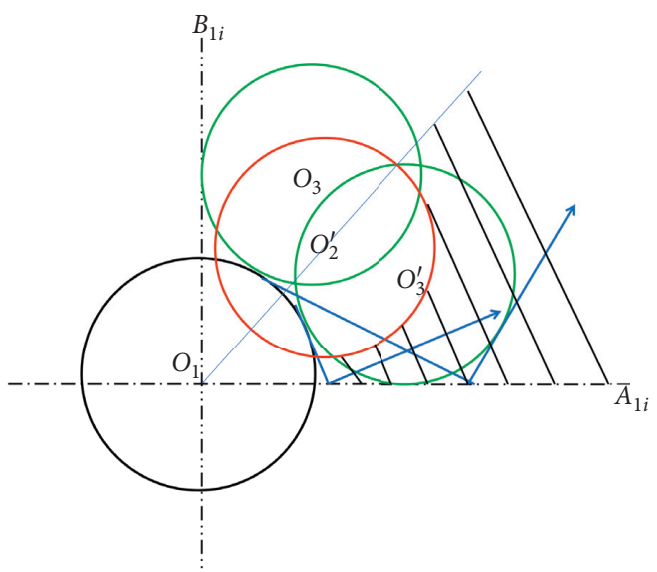

(b)

FIgURE 5: The position diagram of the simplified model and the real model.

The steps for combining the Levenberg-Marquardt algorithm and the Armijo algorithm are as follows:

Step 1. Use the intersection point in Figure 5 as the current initial value $x_{0}$. Compute $n=$ length $\left(x_{0}\right)$; length is a function that calculates the length of an array dimension, and give the allowed error $\delta$.

Step 2. Compute the function $f_{k}=\operatorname{feval}\left(F_{k}, x_{0}\right)$, muk $=\operatorname{norm}\left(\operatorname{feval}\left(F_{k}, x_{0}\right)\right)$, where feval is execute the specified function and norm is matrix or vector norm function.

Step 3. Compute the Jacobian matrix: $j f_{k}=$ feval $\left(j F_{k}, x_{0}\right)$.

Step 4. Compute $g k=j f_{k}^{\prime} * f_{k}$.

Step 5. Solve a system of equations: $\mathrm{d} k=-\left(j f_{k}^{\prime} *\right.$ $\left.j f_{k}+m u k * E(n)\right) / g k$, where $E$ is the identity matrix function.

Step 6. If norm $(g k)<\delta$, then go to step 9; using the Armijo algorithm, find the step size.

Step 7. Given the initial value $\varepsilon \in(0,1), \oplus \in(0,0.5)$, $m_{k}=0$, where $k$ is the maximum number of iterations.

Step 8. If the inequality $f\left(x_{k}+\alpha_{k} d_{k}\right) \leq f\left(x_{k}\right)+$ $\alpha_{k} \oplus G^{T} d_{k}$ is true, then $m_{k}^{\prime}=m_{k}, x_{k+1}=x_{k}+\varepsilon^{m_{k}^{\prime}} d_{k}$, and stop calculation; else, go to step 9, where $d_{k}$ is the search direction, $\varepsilon^{m_{k}^{\prime}}$ is the step size factor, and $\alpha_{k}=\beta^{m_{k}}$.

Step 9. $m_{k}=m_{k}+1$; go to step 7 .

Step 10. If the contact deformation $\lambda_{1 i}>0, \lambda_{2 i}>0$, then stop calculation; else return to the first-layer model to adjust the initial value by the initial value search policy.

Remark 2. The bilevel model for the quasistatic solution of ball bearings is established. The Levenberg-Marquardt algorithm and the Armijo algorithm are adopted to solve the model, which transforms the problem of solving the nonlinear equations into an optimization problem.
3.3. Numerical Analysis and Calculation of the Bilevel Solution Algorithm Model. According to the above description, a bilevel model for the quasistatic solution of ball bearings is established. The process of the layered $j$ iteration is shown in Figure 6.

\section{Results and Discussion}

4.1. Type Selection of the Bearing. An ACBB 71808 bearing is selected as the single-layer ball bearing, and the specific size parameters are shown in Table 1.

The inner layer bearing of the double-decker ball bearing is designated as 71901C, the outer layer bearing is designated as $71905 \mathrm{C}$, and the specific parameters are shown in Table 2.

4.2. Verification of the Proposed Method. To verify the correctness of the method, the important parameter of ball bearing of the contact angle is selected as a study case. Figure 7 shows the relationship between the contact angle and the position angle of a single-layer ball bearing using the Newton-Raphson method and the proposed method. As the rotation speed increases, the contact angle between the ball and the outer raceway increases, while the contact angle between the ball and the inner raceway decreases. This trend is consistent with the Newton-Raphson method. The maximum error of the contact angle is $0.84 \%$, and the correctness of the proposed method is proved.

4.3. Results of the Analysis of the Search Data, the Offset Distance, and the Time. The search step, the offset distance, and the time are selected as the evaluation results. The number of searches (not the number of iterations) is defined as the number of the initial value experiments, which attempts to make the program converge and the result correct. It is used to evaluate the finite solution of the new method.

The long distance between the true value and the current value does not mean that the truth value cannot be solved, but the closer the initial value is to the truth value, the higher the probability is that it can be solved correctly. Therefore, 


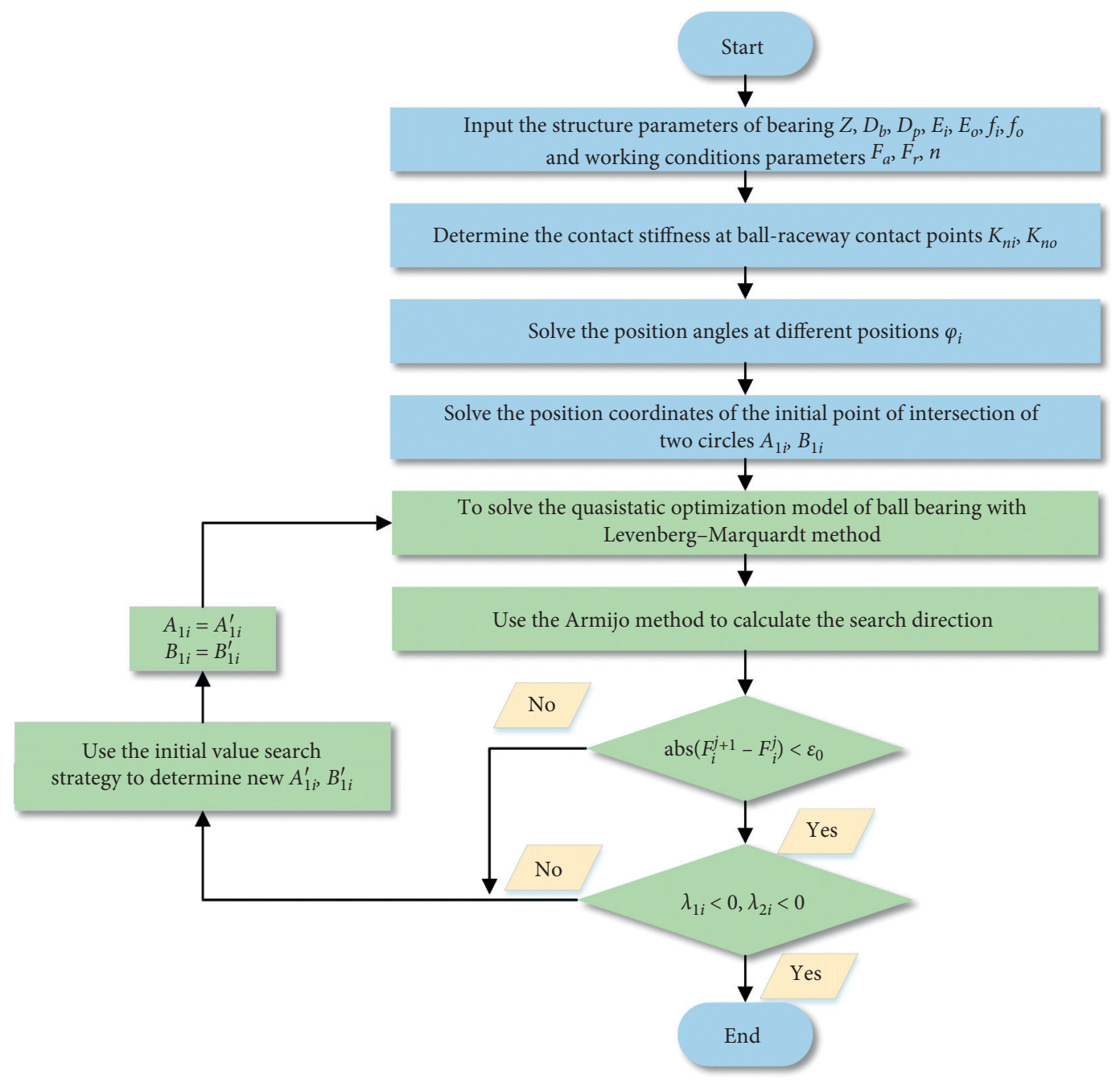

FIGURE 6: Numerical analysis and calculation flowchart of the bilevel model.

TABLE 1: The parameters of the single-layer ball bearing.

\begin{tabular}{lc}
\hline Parameter & Value \\
\hline Diameter of ball $D(\mathrm{~mm})$ & 3.175 \\
Initial contact angle $\alpha_{0}\left({ }^{\circ}\right)$ & 25 \\
Number of ball $Z$ & 29 \\
Groove curvature coefficients of inner raceway $f_{i}$ & 0.515 \\
Groove curvature coefficients of outer raceway $f_{o}$ & 0.525 \\
Pitch diameter $D_{p}(\mathrm{~mm})$ & 46 \\
\hline
\end{tabular}

the offset distance between the current point and the truth value is chosen as another evaluation index, which is used to measure the reliability of the initial value search. The distance deviation is defined as the distance between the position of each search point and the true position, the calculation formula is as follows, where $\left(x_{t}^{\prime}, y_{t}^{\prime}\right)$ is the mean of the current initial value points and $\left(x_{t}, y_{t}\right)$ is the mean of the value of ideal truth points:

$$
\mathrm{I}=\sqrt{\left(x_{t}-x_{t}^{\prime}\right)^{2}+\left(y_{t}-y_{t}^{\prime}\right)^{2}} \text {. }
$$

The time complexity is selected to evaluate the rapidity and stability of the new method.
TABle 2: The parameters of the double-decker ball bearing.

\begin{tabular}{lcc}
\hline \multirow{2}{*}{ Parameter } & \multicolumn{2}{c}{ Value } \\
& $71901 \mathrm{C}$ & $71905 \mathrm{C}$ \\
\hline Diameter of ball $(\mathrm{mm})$ & 3 & 4.25 \\
Initial contact angle $\left(^{\circ}\right)$ & 15 & 15 \\
Number of ball & 17 & 21 \\
Groove curvature coefficients of inner raceway & 0.515 & 0.525 \\
Groove curvature coefficients of outer raceway & 0.515 & 0.525 \\
Pitch diameter $(\mathrm{mm})$ & 18 & 33.5 \\
\hline
\end{tabular}

4.3.1. Single-Layer Ball Bearing. The comparison of the experiment data of the type of 71808 and B218 bearings is shown in Table 3. The experimental environment is Intel Xeon X5650, $2.66 \mathrm{GHz}^{*} 6$ Core, $32 \mathrm{~GB}$ on Windows 10, and MATLAB 2015b. The numerical results under different working conditions are analyzed. Since the angular contact ball bearings mainly bear axial load, the working condition is select under the axial load and rotating speed increases. As the axial load and rotating speed increase, the number of searches slightly increases because the increases in load and speed affect the relationship between the locations of the two rounds, but both converge within a few steps. The 


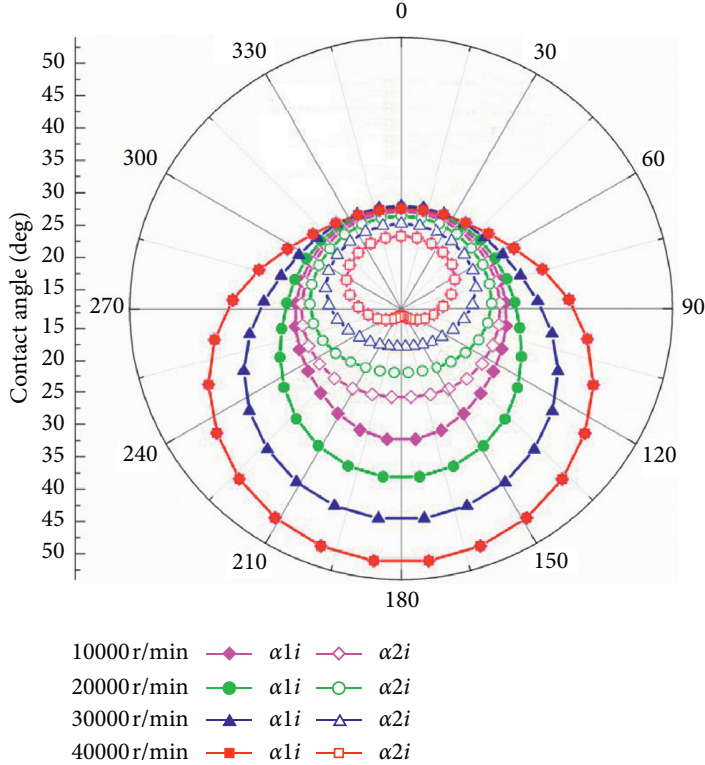

(a)

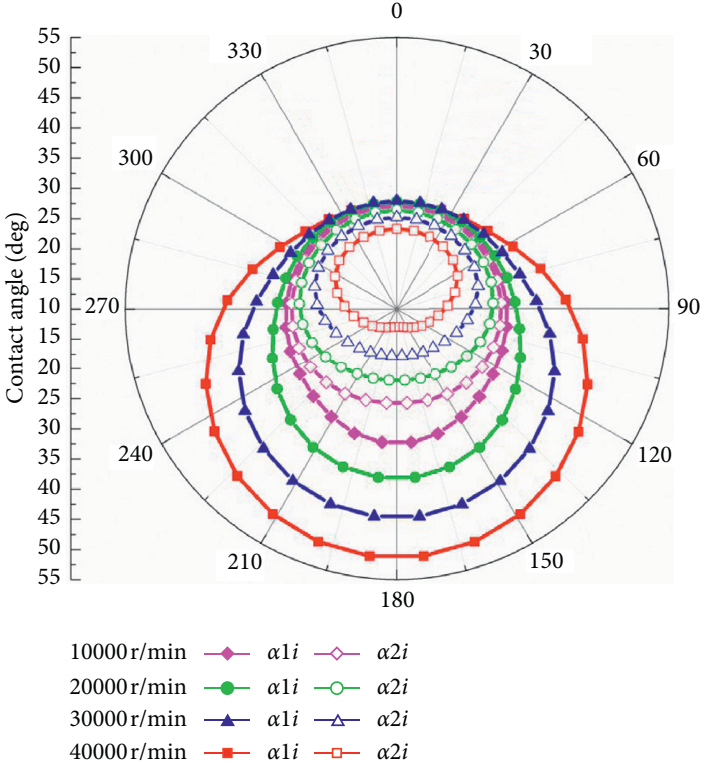

(b)

Figure 7: The comparison of the relationship of the contact angle and position angle between the Newton-Raphson method and the proposed method. (a) By the Newton-Raphson method. (b) By the proposed method.

TABLE 3: The comparison of the search data and the calculation time of a single-layer ball bearing.

\begin{tabular}{|c|c|c|c|c|c|c|c|c|c|c|}
\hline \multirow{2}{*}{$\begin{array}{l}\text { Bearings } \\
\text { type }\end{array}$} & \multirow[b]{2}{*}{ Test } & \multicolumn{3}{|c|}{ Working condition } & \multicolumn{2}{|c|}{ The number of search times } & \multicolumn{2}{|c|}{ Total time (s) } & \multicolumn{2}{|c|}{ The single search time $(\mathrm{s})$} \\
\hline & & $\begin{array}{l}\mathrm{Fr} \\
(\mathrm{N})\end{array}$ & $\begin{array}{c}\mathrm{Fa} \\
(\mathrm{N})\end{array}$ & $\begin{array}{c}n \\
(\mathrm{r} / \mathrm{min})\end{array}$ & $\begin{array}{c}\text { Newton-Raphson } \\
\text { method }\end{array}$ & $\begin{array}{l}\text { Proposed } \\
\text { method }\end{array}$ & $\begin{array}{l}\text { Newton-Raphson } \\
\text { method }\end{array}$ & $\begin{array}{l}\text { Proposed } \\
\text { method }\end{array}$ & $\begin{array}{l}\text { Newton-Raphson } \\
\text { method }\end{array}$ & $\begin{array}{c}\text { Proposed } \\
\text { method }\end{array}$ \\
\hline \multirow{11}{*}{71808} & 1 & 200 & 200 & 10000 & \multirow{24}{*}{$\begin{array}{l}\text { By trying the initial } \\
\text { value manually, the } \\
\text { uncertainty of the } \\
\text { initial value } \\
\text { determines the } \\
\text { uncertainty of the } \\
\text { search times. }\end{array}$} & 3 & \multirow{24}{*}{$\begin{array}{l}\text { By trying the initial } \\
\text { value manually, the } \\
\text { uncertainty of the } \\
\text { initial value } \\
\text { determines the } \\
\text { uncertainty of the } \\
\text { total times. }\end{array}$} & 27.323 & \multirow{24}{*}{$\begin{array}{l}\text { By trying the initial } \\
\text { value manually, the } \\
\text { uncertainty of the } \\
\text { initial value } \\
\text { determines the } \\
\text { uncertainty of the } \\
\text { single search times. }\end{array}$} & 9.1077 \\
\hline & 2 & 200 & 200 & 20000 & & 3 & & 29.641 & & 9.8803 \\
\hline & 3 & 200 & 200 & 30000 & & 4 & & 40.126 & & 10.0315 \\
\hline & 4 & 200 & 200 & 40000 & & 5 & & 48.753 & & 9.7506 \\
\hline & 5 & 200 & 400 & 10000 & & 3 & & 28.142 & & 9.3807 \\
\hline & 6 & 200 & 400 & 20000 & & 3 & & 28.97 & & 9.6567 \\
\hline & 7 & 200 & 400 & 30000 & & 5 & & 49.211 & & 9.8422 \\
\hline & 8 & 200 & 400 & 40000 & & 7 & & 60.094 & & 8.5849 \\
\hline & 9 & 200 & 600 & 10000 & & 3 & & 28.639 & & 9.5463 \\
\hline & 10 & 200 & 600 & 20000 & & 3 & & 29.785 & & 9.9283 \\
\hline & 11 & 200 & 600 & 30000 & & 7 & & 62.193 & & 8.8847 \\
\hline \multirow{13}{*}{ B218 } & 12 & 200 & 600 & 40000 & & 8 & & 70.516 & & 8.8145 \\
\hline & 13 & 500 & 1000 & 0 & & 3 & & 26.763 & & 8.9210 \\
\hline & 14 & 500 & 1000 & 5000 & & 5 & & 35.267 & & 7.0534 \\
\hline & 15 & 500 & 1000 & 10000 & & 7 & & 56.726 & & 8.1037 \\
\hline & 16 & 500 & 1500 & 0 & & 3 & & 29.878 & & 9.9593 \\
\hline & 17 & 500 & 1500 & 5000 & & 6 & & 47.252 & & 7.8753 \\
\hline & 18 & 500 & 1500 & 10000 & & 7 & & 59.646 & & 8.5209 \\
\hline & 19 & 1000 & 2000 & 0 & & 3 & & 27.514 & & 9.1713 \\
\hline & 20 & 1000 & 2000 & 5000 & & 4 & & 36.582 & & 9.1455 \\
\hline & 21 & 1000 & 2000 & 10000 & & 6 & & 46.913 & & 7.8188 \\
\hline & 22 & 1000 & 3000 & 0 & & 3 & & 31.122 & & 10.3740 \\
\hline & 23 & 1000 & 3000 & 5000 & & 7 & & 60.537 & & 8.6481 \\
\hline & 24 & 1000 & 3000 & 10000 & & 8 & & 67.128 & & 8.3910 \\
\hline
\end{tabular}

calculation results show that the new method can effectively decrease the number of search implementations, realizing efficient and finite solutions.
The fast and stable performance of the new method is evaluated by the total time and the single search time, respectively. In the design experiment in Table 3, the total time 
for solving in each condition ranges from $26.763 \mathrm{~s}$ to $67.128 \mathrm{~s}$, while the total solving time of the traditional Newton-Raphson method is uncertain, which shows that the new method can achieve finite and fast solution. What is more, the single search time is from 7.0534s to $10.0315 \mathrm{~s}$; it is proved that the computational complexity of the new method is acceptable and stable.

Figures 8 and 9 show the offset distance graphs corresponding to different search steps in the single-layer bearing tests with models 71808 and B218. It can be seen from the figure that although each test has different convergence steps, the offset distance of the search gradually decreases and approaches the truth value gradually, indicating that the search direction is correct and the method is stable and reliable.

4.3.2. Double-Decker Bearing Model. The proposed method is extended to calculate the double-decker bearings. The number of nonlinear equations of solution for doubledecker bearing is $4 \mathrm{Z}+4$. Figure 10 shows the relation of the contact angle and position angle of the double-decker ball bearing. When the speed is not equal to zero, the ball produces centrifugal force, and the contact angles between the inner and outer raceways and the ball are unequal. The contact angles between the inside and outside layers of the ball bearings and the inner raceway are both increased, and the contact angle of the ball bearing with the outer raceway is reduced because of the effect of the double-decker bearing speed distribution ratio. The influence of the centrifugal force of the external layer of the ball bearing on the contact angle is less than that of the centrifugal force of the inner layer of the ball bearing and the influence of the internal and external contact angles. As a result, the change in the outer bearing contact angle range is not large. The maximum error of the contact angle between the Newton-Raphson method and the proposed method is $1.1 \%$; the calculation results illustrate the correctness of the method.

The search data, the total time, and the single search time of the double-decker bearing are shown in Table 4. The new proposed method can search the adjacent initial values in a few attempts. The search data and the total time perform longer than that of single-layer bearing because the parameters of double-decker bearing are twice as much as that of the single-layer bearing. But they are all solved in a limited time ranged from $90.143 \mathrm{~s}$ to $150.187 \mathrm{~s}$. The single search time is from $15.0238 \mathrm{~s}$ to $18.8521 \mathrm{~s}$. It is shown that the method is stable and acceptable for the solution of doubledecker bearing. The proposed method effectively solves the problem of manually randomly trying different initial values and searching without a range, and the high efficiency and finite solution of the double-decker bearing are realized.

Figure 11 shows the offset distance diagram corresponding to different search steps in the double-decker bearing test. As can be seen from the figure, with the increase of search steps, the offset distance gradually decreases and

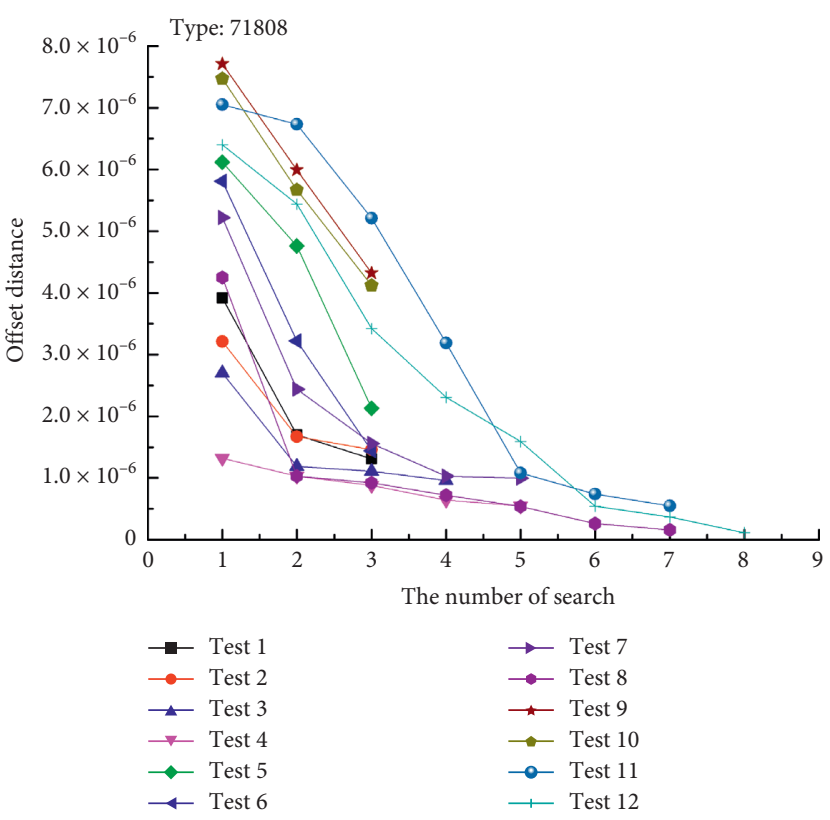

Figure 8: The offset distance of type of 71808.

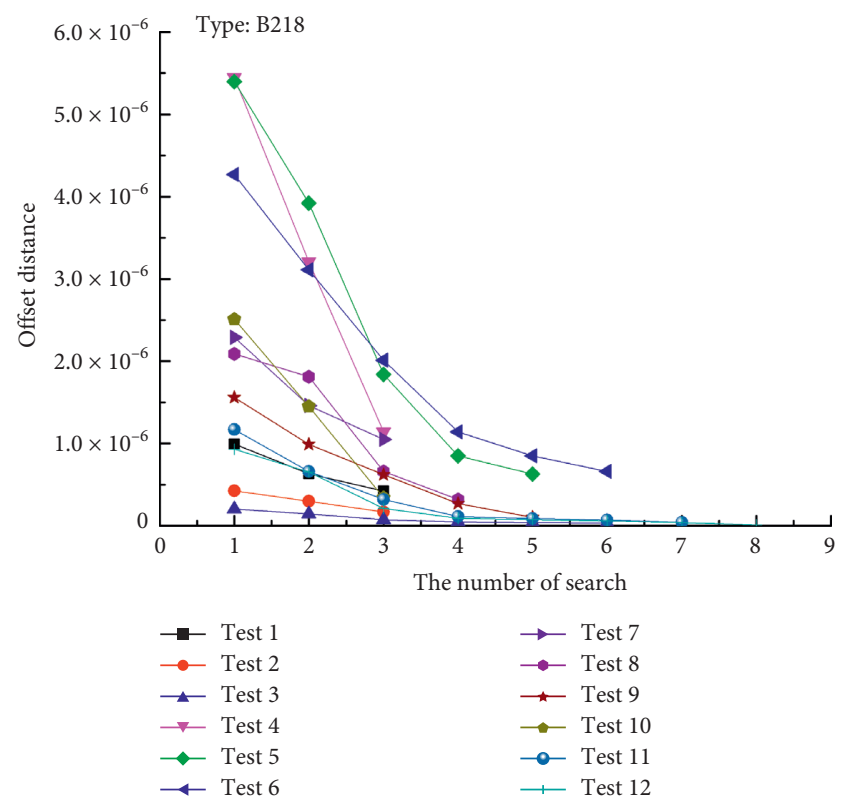

Figure 9: The offset distance of type of B218.

gets closer to the truth value, indicating that the search direction is correct and proving that the method is stable and reliable.

Remark 3. The new method can be extended to the calculation of the double-decker ball bearings, which improves the applicability of the new method and realizes the finite and fast solution of the model. 


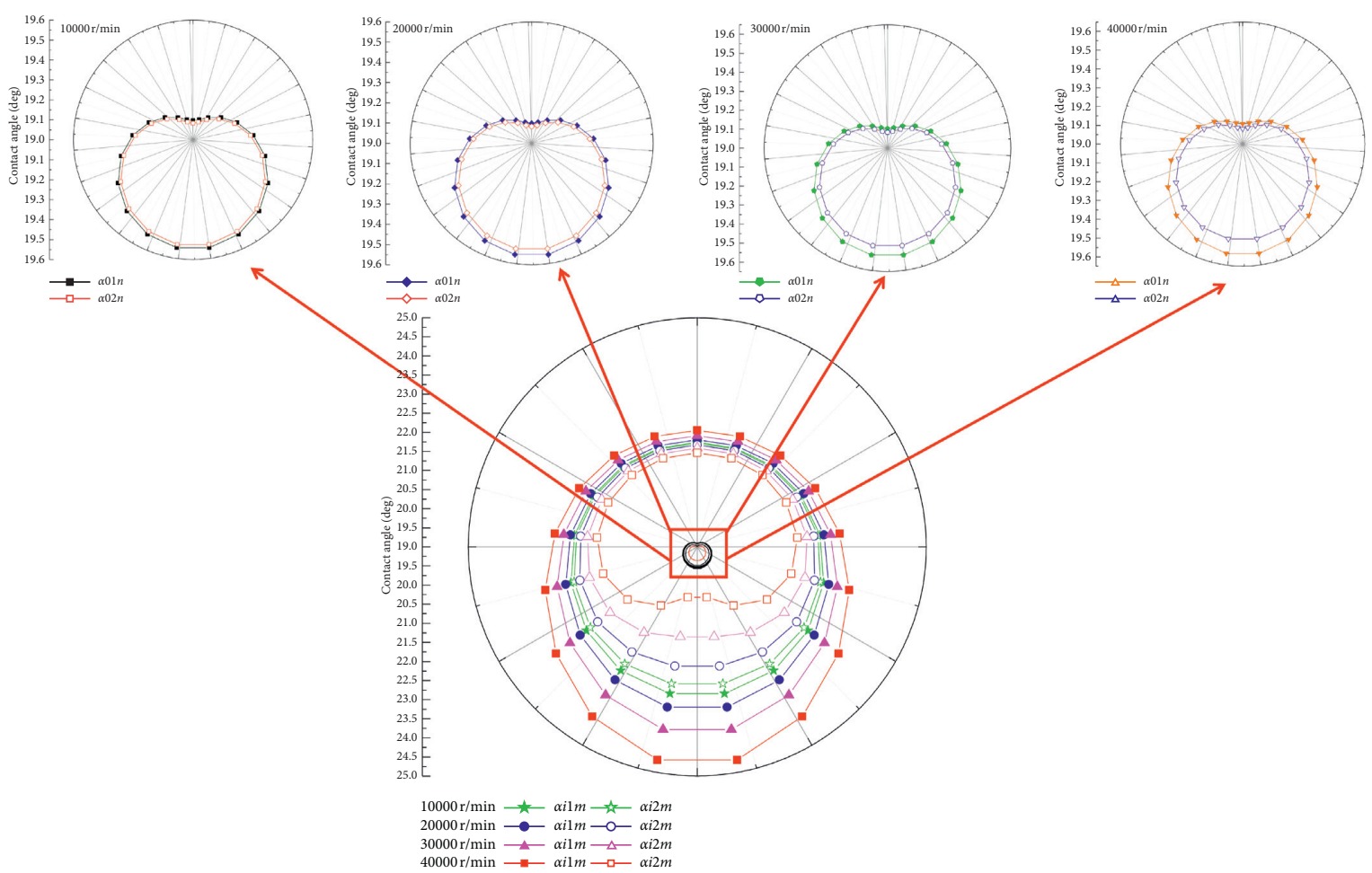

(a)
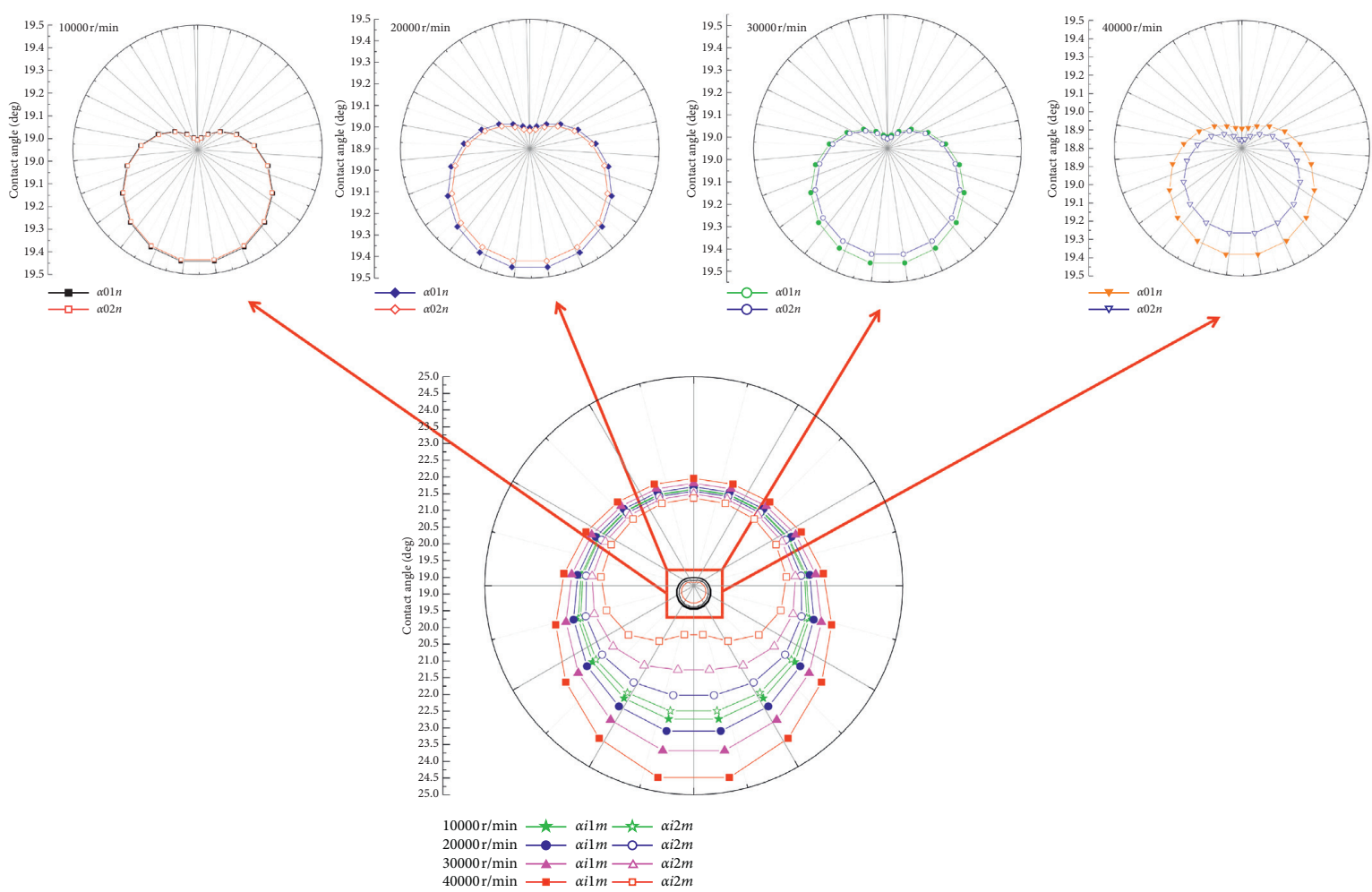

(b)

Figure 10: The comparison of the relationship of the contact angle and position angle of the double-decker ball bearing between the Newton-Raphson method and the proposed method. (b) By the proposed method. (a) By the Newton-Raphson method. 
TABLE 4: The comparison of the search data and the calculation time of the double-decker bearing.

\begin{tabular}{|c|c|c|c|c|c|c|c|c|c|c|}
\hline \multirow{2}{*}{$\begin{array}{l}\text { Bearings } \\
\text { type }\end{array}$} & \multirow{2}{*}{ Test } & \multicolumn{3}{|c|}{$\begin{array}{l}\text { Working } \\
\text { condition }\end{array}$} & \multicolumn{2}{|c|}{ The number of search times } & \multicolumn{2}{|c|}{ Total time (s) } & \multicolumn{2}{|c|}{ The single search time (s) } \\
\hline & & $\begin{array}{l}\mathrm{Fr} \\
(\mathrm{N})\end{array}$ & $\begin{array}{l}\mathrm{Fa} \\
(\mathrm{N})\end{array}$ & $\begin{array}{c}n \\
(\mathrm{r} / \mathrm{min})\end{array}$ & $\begin{array}{l}\text { Newton-Raphson } \\
\text { method }\end{array}$ & $\begin{array}{c}\text { Proposed } \\
\text { method }\end{array}$ & $\begin{array}{l}\text { Newton-Raphson } \\
\text { method }\end{array}$ & $\begin{array}{l}\text { Proposed } \\
\text { method }\end{array}$ & $\begin{array}{l}\text { Newton-Raphson } \\
\text { method }\end{array}$ & $\begin{array}{c}\text { Proposed } \\
\text { method }\end{array}$ \\
\hline \multirow{12}{*}{$\begin{array}{l}\text { 71901C } \\
71905 C\end{array}$} & 1 & 200 & 200 & 10000 & \multirow{12}{*}{$\begin{array}{l}\text { By trying the initial } \\
\text { value manually, the } \\
\text { uncertainty of the } \\
\text { initial value } \\
\text { determines the } \\
\text { uncertainty of the } \\
\text { search steps. }\end{array}$} & 6 & \multirow{12}{*}{$\begin{array}{l}\text { By trying the initial } \\
\text { value manually, the } \\
\text { uncertainty of the } \\
\text { initial value } \\
\text { determines the } \\
\text { uncertainty of the } \\
\text { total times. }\end{array}$} & 90.143 & \multirow{12}{*}{$\begin{array}{l}\text { By trying the initial } \\
\text { value manually, the } \\
\text { uncertainty of the } \\
\text { initial value } \\
\text { determines the } \\
\text { uncertainty of the } \\
\text { single search times. }\end{array}$} & 15.0238 \\
\hline & 2 & 200 & 200 & 20000 & & 7 & & 108.636 & & 15.5194 \\
\hline & 3 & 200 & 200 & 30000 & & 7 & & 112.581 & & 16.0830 \\
\hline & 4 & 200 & 200 & 40000 & & 8 & & 131.64 & & 16.4550 \\
\hline & 5 & 200 & 400 & 10000 & & 6 & & 92.591 & & 15.4318 \\
\hline & 6 & 200 & 400 & 20000 & & 7 & & 115.74 & & 16.5343 \\
\hline & 7 & 200 & 400 & 30000 & & 8 & & 136.219 & & 17.0274 \\
\hline & 8 & 200 & 400 & 40000 & & 8 & & 137.415 & & 17.1769 \\
\hline & 9 & 200 & 600 & 10000 & & 7 & & 106.173 & & 15.1676 \\
\hline & 10 & 200 & 600 & 20000 & & 7 & & 128.318 & & 18.3311 \\
\hline & 11 & 200 & 600 & 30000 & & 8 & & 149.363 & & 18.6704 \\
\hline & 12 & 200 & 600 & 40000 & & 8 & & 150.817 & & 18.8521 \\
\hline
\end{tabular}

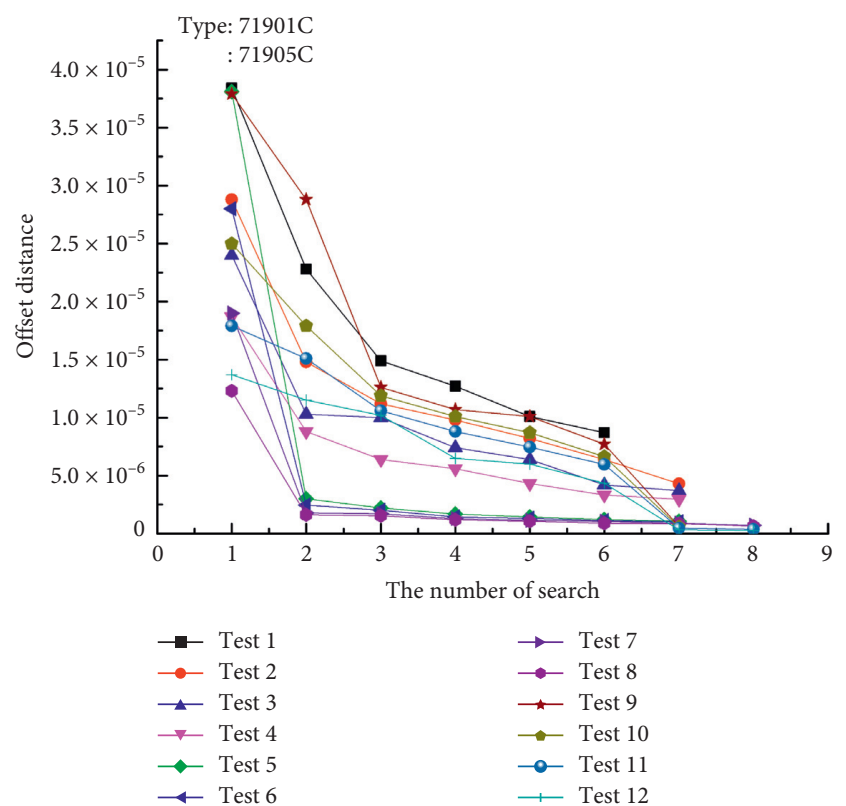

FIGURE 11: The offset distance of the double-decker ball bearing.

From the contrast case which can be seen in the example, the proposed method can solve the problem of the quasistatic analysis of the ball bearing. The result is proved by a study case; however, unlike the traditional methods, the bilevel model is established. From the geometric relationship of the ball bearing of numerical analysis, this paper puts forward the initial value search policy. By converting the uncertain problems to a certain problem, it thus reduces the artificial trying of the initial value by experience. So, the new method can solve the problem in a finite number of steps.

\section{Conclusion}

In this paper, based on a quasistatic analysis of ball bearings, a new method for finite initial value optimization is proposed. First, a bilevel model is established, and an initial value search strategy is proposed; the adjacent reasonable

initial value can be found in a few steps, which compensates for the shortcoming of searching the initial value manually. Then, the Levenberg-Marquardt algorithm and the Armijo algorithm were used to solve the optimization problem. The fast solution of quasistatic analysis of ball bearings is realized. The method can be extended to the calculation of the double-decker bearings. Finally, the correctness of the new method is proved by comparison with the traditional method and numerical analysis experiment. In future work, it will be improved to calculate the multi-DOF conditions, and the proposed methods can provide a basis for the rapid solution of multi-DOF models in the future.

\section{Abbreviations}

$Z, Z_{i b}, Z_{o b}$ :

$D_{b}, D_{i b}, Z_{o b}$ :

$D_{p}, D_{i p}, D_{o p}$

$\alpha_{0}, \alpha_{i 0}, \alpha_{o 0}$ :

$f_{i}, f_{i 1}, f_{i 2}, f_{o}$,

$f_{o 1}, f_{o 2}$ :

$\varphi_{i}, \varphi_{i m}, \varphi_{o n}:$

$E_{i}, E_{o}$ :

$\Delta_{r}, \Delta_{i r}, \Delta_{o r}, \Delta_{a}, \Delta_{i a}$

$\Delta_{o a}$ :

$\lambda_{1 i}, \lambda_{i 1 m}, \lambda_{i 2 m}, \lambda_{2 i}$,

$\lambda_{o 1 n}, \lambda_{o 2 n}:$

$\lambda_{r}, \lambda_{i r}, \lambda_{o r}, \lambda_{a}, \lambda_{i a}$,

$\lambda_{o a}$ :

$X, X_{i}, X_{o}$ :

$A_{1 i}, B_{1 i}, A_{i 1 m}, B_{i 1 m}$, $A_{o 1 n}, B_{o 1 n}$ :

$A_{2 i}, B_{2 i}, A_{o 2 n}, B_{o 2 n}$,

$A_{i 2 m}, B_{i 2 m}$ :

$\alpha_{1 i}, \alpha_{2 i}, \alpha_{i 1 m}, \alpha_{i 2 m}$,

$\alpha_{o 1 n}, \alpha_{o 2 n}$ :
Ball number

Ball diameter ( $\mathrm{mm}$ )

Pitch diameter ( $\mathrm{mm}$ )

Initial contact angle $\left({ }^{\circ}\right)$

Radius coefficient of curvature of inner/outer groove

Position angle

Modulus of elasticity

Radial clearance and axial clearance

(mm)

Contact deformation ( $\mathrm{mm}$ )

Radial and axial displacement of the inner ring $(\mathrm{mm})$

Distance between the center of curvature of the raceway when not subjected to load ( $\mathrm{mm}$ )

Axial projection and radial projection between the center of curvature of the outer rings and ball center

Axial and radial projections between the curvature center of the inner and outer rings

Inner and outer contact angle $\left({ }^{\circ}\right)$ 


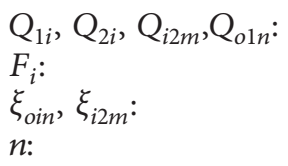

Contact force $(\mathrm{N})$

Centrifugal force $(\mathrm{N})$

Guidance coefficient

Speed (r/min).

\section{Data Availability}

The data used to support the findings of this study are available from the corresponding author upon request.

\section{Conflicts of Interest}

The authors declare that they have no conflicts of interest regarding the publication of this paper.

\section{Acknowledgments}

This research was supported by the Jilin Province Science and Technology Development Program of China under Contract no. 20170204008GX.

\section{References}

[1] C. J. Zhao, "Analysis on the load characteristics and coefficient of friction of angular contact ball bearing at high speed," Tribology International, vol. 87, pp. 50-56, 2015.

[2] W.-Z. Wang, L. Hu, S.-G. Zhang, Z.-Q. Zhao, and S. Ai, "Modeling angular contact ball bearing without raceway control hypothesis," Mechanism and Machine Theory, vol. 82, pp. 154-172, 2014.

[3] Y. Wu, H. Yan, S. Li, K. Zhang, and L. Zhang, "Calculation on the radiation noise of ceramic ball bearings based on dynamic model considering nonlinear contact stiffness and damping," Journal of Sound and Vibration, vol. 479, p. 115374, 2020.

[4] R. Stribeck, "Ball bearing for various loads," Transactions of the ASME, vol. 29, pp. 420-463, 1907.

[5] A. B. Jones, "Ball motion and sliding friction in ball bearings," Journal of Basic Engineering, vol. 81, no. 1, pp. 1-12, 1959.

[6] T. A. Harris and M. H. Mindel, "Rolling element bearing dynamics," Wear, vol. 23, no. 3, pp. 311-337, 1973.

[7] P. K. Gupta, Advanced Dynamics of Rolling Elements, Springer, Berlin, Germany, 1984.

[8] C. Li, "A new fatigue damage accumulation rating life model of ball bearings under vibration load," Industrial Lubrication and Tribology, 2020.

[9] X. Zhang, H. Xu, W. Chang, H. Xi, and S. N. Xu, "A dynamic contact wear model of ball bearings without or with distributed defects," 2020.

[10] J. Q. Wu, "Investigation on the angular contact ball bearings under low speed and heavy load with coupled mixed lubrication and quasi-dynamic analysis," Lubrication Science, 2020.

[11] X.-J. Shi, L.-Q. Wang, Y.-Z. Mao, and F.-Q. Qin, “Coupling study on dynamics and TEHL behavior of high-speed and heavy-load angular contact ball bearing with spinning," Tribology International, vol. 88, pp. 76-84, 2015.

[12] J. Hu, Q. Y. Lv, X. M. Zhang, Z. Y. Wei, and H. L. Li, "Finite element analysis of layered, variable density, ball bearings based on pearl and turtle shell bionic structures," Industrial Lubrication and Tribology, 2020.

[13] Y. Q. Liu, W. Z. Wang, H. Liang, and T. Qing, "Nonlinear dynamic behavior of angular contact ball bearings under microgravity and gravity," International Journal of Mechanical Sciences, vol. 183, 2020.

[14] N. T. Liao and J. F. Lin, "Ball bearing skidding under radial and axial loads," Mechanism and Machine Theory, vol. 37, pp. 91-113, 2002.

[15] N.-T. Liao and J. F. Lin, "Rolling-sliding analysis in ball bearing considering thermal effect," Tribology Transactions, vol. 49 , no. 1 , pp. 1-16, 2006.

[16] A. Leblanc, D. Nelias, and D. Plona, "Ball motion and sliding friction in a four-contact-point ball bearing," Journal of Tribology, vol. 129, no. 4, pp. 801-808, 2007.

[17] Z. Wen, Z. Q. Wang, and S. Y. Zhao, "Modeling angular contact ball bearing without raceway control hypothesis," Mechanism and Machine Theory, vol. 82, pp. 154-172, 2014.

[18] J. H. Zhang, B. Fang, J. Hong, and Y. S. Zhu, "Effect of preload on ball-raceway contact state and fatigue life of angular contact ball bearing," Tribology International, 2017.

[19] H. P. Yan, Y. H. Wu, S. H. Li, L. X. Zhang, and K. Zhang, "Research on sound field characteristics of full-ceramic angular contact ball bearing," Journal of the Brazilian Society of Mechanical Sciences and Engineering, 2020.

[20] W. J. Anderson, "Tribology for aerospace application," 1973.

[21] H. Prashad, "A new generation double decker high precision rolling element bearing-concept, development and investigations," Tribology Transactions, vol. 44, no. 2, pp. 203-208, 2001.

[22] C. Yu, L. Xu, and X. Yu, "Research on the mechanical properties of "Z" type double-decker ball bearings," Journal of Tribology, vol. 136, pp. 111021-111028, 2014.

[23] Y. Zhu, Y. Zhang, and C. Jin, "Research on the mechanical properties of a new "I" type double-decker ball bearing," Journal of Tribology, vol. 138, 2016.

[24] L. Q. Wang, Design and Numerical Analysis of Rolling Elements Bearing for Extreme Applications, Harbin Institute of Technology Press, Harbin, China, 2013.

[25] Y. Zhang, G. Sun, T. C. Lim, and L. Xie, "A fast and reliable numerical method for analyzing loaded rolling element bearing displacements and stiffness," Journal of Vibro Engeineering, vol. 17, pp. 620-642, 2015.

[26] W. Deng, J. J. Xu, Y. J. Song, and H. M. Zhao, "Differential evolution algorithm with wavelet basis function and optimal mutation strategy for complex optimization problem," Applied Soft Computing Journal, 2020.

[27] W. Deng, J. J. Xu, X. Z. Gao, and H. M. Zhao, "An enhanced MSIQDE algorithm with novel multiple strategies for global optimization problems," IEEE Transactions on Systems Man Cybernetics-Systems, 2020.

[28] X. Li, k. Yu, H. Ma et al., "Analysis of varying contact angles and load distributions in defective angular contact ball bearing," Engineering Failure Analysis, vol. 91, pp. 449-464, 2018.

[29] Y. L. Zhu and L. X. Xu, "The radial stiffness and application of double-decker ball bearing," Key Engineering Materials, vol. 450, pp. 353-356, 2011.

[30] W. Deng, J. J. Xu, H. M. Zhao, and Y. J. Song, "A novel gate resource allocation method using improved PSO-based QEA," IEEE Transactions on Intelligent Transportation Systems, 2020.

[31] W. Deng, H. L. Liu, J. J. Xu, H. M. Zhao, and Y. J. Song, “An improved quantum-inspired differential evolution algorithm for deep belief network," Transactions on Instrumentation and Measurement, 2020.

[32] H. Mirzaee, "Long-term prediction of chaotic time series with multi-step prediction horizons by a neural network with 
Levenberg-Marquardt learning algorithm," Chaos, Solitons \& Fractals, vol. 41, no. 4, pp. 1975-1979, 2009.

[33] C.F. Ma, Optimization Method and Matlab Program Design, Science Press, Beijing, China, 2010.

[34] M. Ahookhosh, K. Amini, and S. Bahrami, "A class of nonmonotone Armijo-type line search method for unconstrained optimization," Optimization, vol. 61, no. 4, pp. 387-404, 2012.

[35] B. Peng, L. Wang, L. Cui, and L. Ying, "Numerical solution of analysis model for angular-contact ball bearings," Journal of Nanjing University of Aeronautics and Astronautics, vol. 41, pp. 370-374, 2009. 